\title{
An Intelligent Agent for the Resolution of the Problems of Optimization for Mobile Phone Operators
}

\author{
Chaker Mezioud \\ MISC Laboratory \\ Mentouri University of Constantine (Algeria)
}

\author{
Mohamed-Khireddine Kholladi \\ MISC Laboratory \\ Mentouri University of Constantine (Algeria)
}

\begin{abstract}
The current systems of communication know a considerable development thanks to the mobile communication and more particularly the cellular telephony. Reason for which the suppliers of service begin to find the big motivation, and make a commitment to have the means to be invested in enormous infrastructures of network. The conception of big cellular networks is a complex problem, which turns around a big impact: the quality of the service and the cost of the network. With the continuous and fast growth of the traffic of communication, the large-scale planning becomes harder and harder. The progress in this sector will lead certainly to the important improvements about the quality of service and the cost of deployment. The environment of the cellular networks is a fluctuating environment. The request in traffic varies in hour by hour and from a link to the other one. The appeal to a process of design becomes indispensable, the objective of which is to supply networks capable of evolving and at a lower cost. The detailed and comprehensive analysis of the behavior of the traffic in the time and in the space is an indispensable process for the planning of the cellular network. In this subject, we propose the theme of the Multi Agents Systems as the foundation of our work, for purpose to propose an intelligent agent implanted at the level of every cell(zone of the territory lit by an antenna "BTS") and which serves to anticipate the modifications to be operated on the cellular network, to optimize the performances of the network during its evolution and to reduce the costs of its reorganization.
\end{abstract}

\section{Keywords}

GSM, Cellular Networks, Multi Agents Systems, Dynamic Optimization.

\section{INTRODUCTION}

The industry of the communications knew a prodigious development during the last decade. It is developed beyond all the waits. At the same time, the techniques of radio communications dress henceforth a crucial importance for an increasing number of public utilities. It is necessary to be able to determine the technical characteristics and the ways of exploitation of these Hertzian services, always more numerous, while managing in a optimal way the radio spectre [1]. This last one is a limited natural resource which is always the object of more requests because of the fast development of new services of radio communications and of the phenomenal craze for the mobile techniques. The cellular networks are systems with strong factors of tensions [9]. The real-time adjustment of the solution being at present impossible. The dynamic aspect adds a new dimension to the problem of conception (design) of the cellular networks [4]. The optimization serves then to anticipate the modifications to be operated on the network to optimize the performances of the network during its evolution and to reduce the costs of its reorganization. The system not only has to supply a solution, but also be able to react to the breakdowns and to the chances by straightening its solution, or still to be capable of treating problems the data of which are only partially known, and for which the information arrives in time. The detailed and comprehensive analysis of the behavior of the traffic in the time and in the space is an indispensable process for the planning of the systems radio mobiles. The static sizing of the network so as to offer a level of reduced service at the time of point in for effect to waste the resources of the system when these are not well run. Thus, the idea leads us to think about the development of a process of design capable of managing the changes arising on the network during its phases of growth and maturation. The request in traffic varies in the time and in the space. The network is subject, besides, to changes long term, required by the rise of the traffic, relative to the growth of the clientele, to the behavioral changes of the users as well as to the evolution of the cover. For landing in these requirements, the network is brought to adapt itself and to grow. However, according to the point of view of the researchers, the objectives of the supplier of the network and the specific conditions of the application, several models are to be considered, and thus, several approaches are adopted to resolve them. The appeal to a process of dynamic optimization, seems a promising exit.

This process is periodically launched during the points of decision, for objective the preparation of the system for the adaptation to the changes of the current period, while trying to take into account later periods, by predicting the zones where the increase of capacities is required and by realizing the necessary intelligent changes: (example: period of the holidays or the fairs, to strengthen BTS "basic station receiving the calls going and outgoing ", of concerned zones). Because often, these changes lead an additional cost (new installations, hand of work), what prevents the system from adapting itself to the environment. Therefore, it becomes essential to integrate intelligent systems about the structure of the problem, to conceive methods auto mobile adaptive which can react to the shape of the problem and which will allow to resolve the problem in its entirety. Reason for which we meet in front of the need to bring in the multi agents systems. A discipline which is interested in the collective behavior produced by the interactions of several autonomous and flexible entities called agents, which these interactions turn around the cooperation, around the competition or around the coexistence between these agents. We also think that the development of an intelligent agent is a promising exit. The present paper consists of various sections, we begin by placing the context of the networks of the mobile telephony, its problem, and the main concepts which refer to it. In a second section, we present the Multi Agents Systems. We grant a particular interest to the Intelligent Agent, to arrive at the development of the approach proposed for this study. The last section will make the objective of a conclusion and perspectives.

\section{GENERAL STRUCTURE OF A MOBILE NETWORK}

The purpose of a mobile network is to provide a range of telecommunications services to users regardless of their movement within a territory. To this end mobile subscriber uses two distinct elements:

- A mobile equipment or terminal, which supplies the radio and software capabilities necessary to dialogue with the network. 
- A removable SIM card (Subscriber Identity Module), which stores the characteristics of the subscriber and his rights, in particular its international identity.

\subsection{GSM Network}

The architecture of a network GSM can be divided into three sub-systems [11]:

- BSS (Base Station Sub-system): assure and manages the transmissions radio.

- NSS (Network Sub System): understands all the necessary functions for call and management of the mobility.

- OSS (Operation Sub System): allows the operator to exploit his network.

\subsection{The equipments of a network GSM}

We can summarize the various components of the network as follows [6]:

- IMEI equipment (International Mobile Equipment Identity) put in the memory of the mobile during its manufacturing.

- Subscriber IMSI (International Mobile Subscriber Identity) being in the SIM card (Subscriber identity Modulates) of the subscriber.

- System of radio communication is the equipment which assures the cover of the cell and includes: the stations of basic transmission BTS (Base Transmitter Station).

- Controller of basic stations BSC (Base Station Controller) which manages between 20 and 30 BTS and possesses its subscribers' register visitors VLR storing the information of the subscriber connected to his mobility.

- Switch of mobile services MSC is an auto switch which assures the necessary functions of switching by directing the conversations to the MSC of the correspondent or to the other networks (phone, Internet) through appropriate interfaces.

- $\quad$ Register of the nominal subscribers or HLR (Home Local Register) is a database used for the management of the mobile subscribers and containing two types of information.

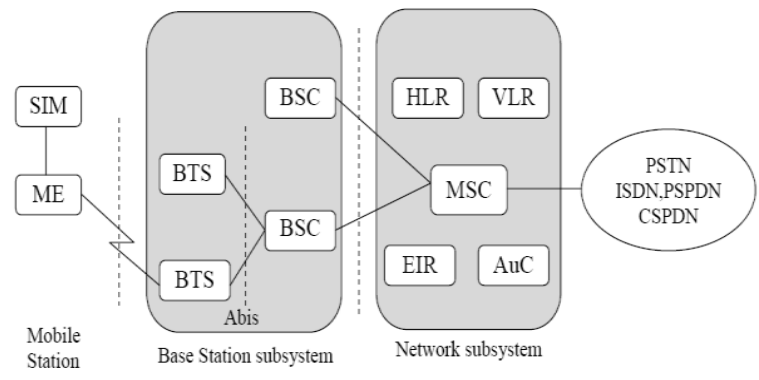

Fig 1. General architecture of the Mobile Network GSM

\subsection{Material architecture of subsystem radio BSS}

BSS includes the BTS which are transmitter-receivers, but having a minimum of intelligence, and the BSC assures the control of a set of BTS.

\subsubsection{Function of BTS}

BTS is a set of transmitter-receivers called TRX. One BTS has for function the management of the transmissions radio (modulation, demodulating, equalization, coding and correction of errors). It also manages the layer connection of data for the exchange of road marking between mobiles and network infrastructure of the operator. So one BTS can manage at most hundred of simultaneous communications.

\subsubsection{Function of BSC}

BSC controls one or several BTS. It has a relay role for the alarms and the statistics emanating from BTS towards the center of exploitation and from maintenance. The BSC is a data bank for software versions and data of configuration downloaded by the operators on BTS. The BSC pilots transfers between two cells (zone of the territory lit by an antenna "BTS"): it informs on one hand new BTS which is going to take care of the subscriber " the mobile " while informing the back-end system (HLR) of the new localization of the subscriber.

\subsection{Material architecture of subsystem fixed NSS}

The NSS includes databases and switches.

\subsubsection{Function of the HLR}

HLR is a database of localization and characteristics of the subscribers. It is considered as recorder of nominal localization by opposition to the VLR which is the recorder of localization of the visitors.

\subsubsection{Function of the MSC}

The MSC assures the interconnection between the mobile network and the fixed network. It manages the establishment of the communications between a mobile and another MSC.

> The handover is a mechanism favor to which a mobile can transfer its connection of one BTS towards the other one, who is in that case called " to handover inter BTS ".

$>$ Or on same BTS of a channel radio towards the other one " handover intra BTS".

\subsubsection{Function of the VLR}

VLR has for mission the recording of the dynamic information relative to the subscribers of passage in the network, so the operator can know all the time in which cell is each of his subscribers. The update of the HLR is very important because when the network tries to join a subscriber, he always questions the HLR of the subscriber to know his last localization.

\subsection{Material architecture of subsystem of exploitation and maintenance OSS}

This subsystem has five main functions:

- The commercial administration.

- The management of the safety.

- The exploitation and the management of the performances.

- The control of configuration of the system.

- The maintenance.

\subsubsection{Function of the AUC}

AUC memorizes for every subscriber a secret key used to authenticate the requests of services and to code the communications.

\subsubsection{Function of the OMC}

OMC is loaded with the local supervision of equipments (BSC, MSC, VLR). Various OMC assure a function of mediation. 


\subsection{Cellular telephony}

A system of radio telephony aims at allowing a terminal to reach the telephone network on a territory of a rather big area (ex: geographical zone). This service uses a radio connection between the terminal and the network. The cellular telephony is a particular case of the radio telephony. A network is said cellular if it includes a set of cell, the size of which depend of the power of emission of issuers and especially the nature of the geographical zone [7].

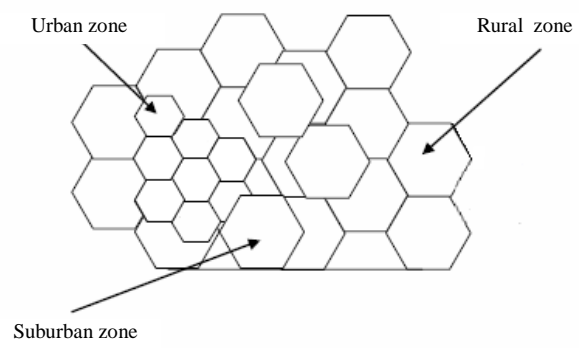

Fig 2. The Cellular Concept

The basic concept of a cellular network is on one hand the division of the territory a set of spaces called cells and on the other hand the sharing of channels radio between cells at the end to avoid the interferences. The size of a cell varies according to a set of constraints among which we find: the relief of territory (plain, mountain), the localization (urban, rural, suburban), the density of subscribers, the nature of the constructions (houses, detached houses, tours) etc.

We can so distinguish several types of cells:

- Macro cells: they are important cells of size going to $35 \mathrm{~km}$, adapted to the weakly populated rural zones. Seen their size, issuers have to supply with very big powers.

- Micro cells: adapted to the dense urban zones, they are cells of very small sizes of some hundreds of meters of beam. They allow to sell a traffic mattering by unity of surface.

- Pico cells: of beam of some tens of meter and adapted to the distribution inside buildings.

We think that the integration of the Multi Agents System will allow us to conceive methods auto adaptive capable of reacting to the shape of the problem. This last idea will make the objective of the next section.

\section{MULTI AGENTS SYSTEMS}

The theme of the Multi Agents Systems (SMA), if it is not recent, are at present a very active field of research. This discipline is for the connection of several domains in particular of the artificial intelligence, the distributed computer systems and the software engineering. It is the discipline which is interested in the collective behavior produced by the interactions of several called autonomous and flexible entities: "agents", whom these interactions turn around the cooperation, around the competition or around the coexistence between these agents.

\subsection{Definition of an agent}

According to an increasing number of researchers defines an agent, as being a computer system situated in an environment [10], of which it is capable of acting in perfect autonomy on its actions, with the aim of making meet the objectives of its conception, with the following characteristics:

$\checkmark$ An agent is an entity situated in a particular environment, which perceives the state of the environment through his sensors, and which acts on its environment through its effectors.

$\checkmark$ It is conceived to achieve specific objectives, and it has particular purposes to be reached.

$\checkmark$ It is autonomous, since it has the control of its internal state, and its own behavior.

$\checkmark$ It is capable of exposing and of producing solutions for flexible problems. It needs to be at once reagent (capable of answering opportunities, changes which arise in the environment and proactive, it is capable of acting by anticipating the future purposes).

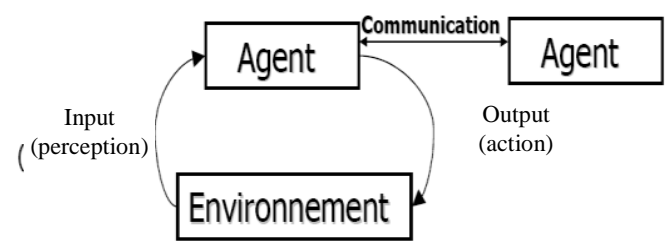

Fig 3. Agent and Environment

\subsection{Characteristics of agents}

The researchers in artificial intelligence agree on the necessity of the existence of some characteristics so that we can speak about an agent. We quote the most essential:

- Autonomy: acts without intervention extern.

- Interaction: communicate with the other agents.

- Pro activity: directed objective, pursues an end.

- Cooperation: capable of coordination with the other agents to reach a common objective.

- Mobility: capable of moving towards another environment [3].

- Rationality: capable of acting according to its internal objectives and its knowledge.

- Intelligence: conscious of its reasoning: faiths, objectives, plans, hypotheses are explicit.

\subsection{Intelligent agent}

There is no universal definition for this type of agent. It adapts its behavior to its environment and by memorizing its experiences, behaves as one under system capable of learning. We can better present it through:

Main features:

$\checkmark$ A computer system (software andlor material).

$\checkmark$ Situated in an environment.

$\checkmark$ Capable of autonomous action in this environment to satisfy its objectives of conception.

"Intelligence" = flexibility of the behavior

$\checkmark \quad$ Ability to react with regard to the environment.

$\checkmark$ Pro-activity: behavior aiming at the realization of purposes social

$\checkmark$ Capacity: cooperation with the other agents (software or human beings) to realize the purposes.

\subsection{Different types of interactions}

The interaction is the mechanism which returns set existing agents in the more dynamic system, by the fact than it brings to light the mechanisms of communication and cooperations [12]. With the means of the interaction all the entities plunged into an environment, can interact according to various forms: 


\subsubsection{Interaction without communication}

It bases itself on the inference of the actions of the others.

Example:

- Use of the theory of the games with matrices of earnings.

- Updating of a constraint or an updating of a dependence.

\subsubsection{Interaction via communication}

It is a set finished of signals without interpretation and having fixed syntax

Example: multi-planning agents.

Example: communication by the environment via tracks (signals) which leave the agents and who can be perceived by the other agents.

\subsubsection{Interaction via sending of messages and plans}

The interaction is made by sending of messages, such as appeals of methods of object-oriented languages.

In the sending of plans, exchanges of partial plans are made so that one a knot of exchange of the partial plans of interpretation with the other knots of the system.

\subsubsection{Interaction via the blackboard}

The interaction via a blackboard, is a technique which proved its efficiency in the conception of the systems, and this used type of technique so that the various existing agents in a given system can to communicate itself through a space called (been divided) common report: "Blackboard", from whom the mechanism and to deposit the information or the knowledge in this zone, the concerned agent will come to get back this information. We introduce this technique of communication, when all the agents is in centralized or distributed architecture. The main characteristics of this type of interaction (blackboard) are the following ones:

- No direct communication.

- Interaction via the sharing of the same space of work or system.

The figure 4 illustrates this mechanism. A system containing a set of agents based on this type of interaction possesses the following elements:

- $\quad$ The knowledge

- The blackboard

- $\quad$ The control mechanism

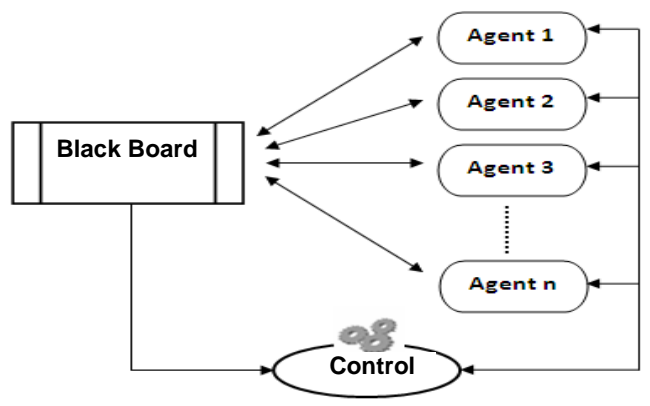

Fig 4. Agents' interaction via the blackboard

The objectives of the next section concern the presentation of our will contribution.

\section{PRESENTATION OF THE APPROACH}

BTS is an element of emission and reception, having a minimum of intelligence, which has for function the management of the transmissions radios. It is the first constituent of the network, responsible on the coverage of the request of a broadcasting subscriber. So that one the other BTS will be informed to take care of the receiving subscriber. The relative load of a cell corresponds to the report between the request in traffic on this cell and its effective capacity. A cell which serves many mobiles sees its coverage area being reduced, thus holes of cover appear and calls will be rejected. To avoid this type of problems, bound to the increase of the traffic, the resource BTS must be exploited in a reliable way (increase its intelligence). Thus; the idea leads us towards the equipment of every BTS by an intelligent, called Agent: Cell Agent.

Cells having a load approaching the $100 \%$ see each other applicants of help of the other BTS neighbors having no load "candidate cells". What can lead an automatic reorganization of the plan of frequencies, without the attribution of new cards TRX nor the insertion of news BTS [2]. A mobile becomes attached to the cell which offers him the best quality radio on the experimental channel (see figure 5: cell with intermittent line).

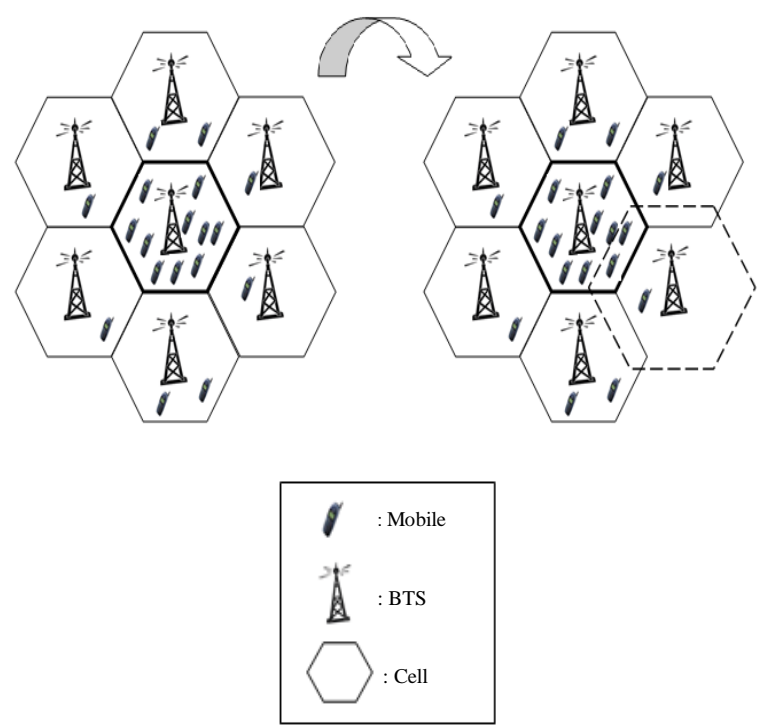

Fig 5. Process of optimization of the cellular network

When a mobile passes from a cell to the other one, he sees the pilot of the first cell weakening, and that of second to grow gradually. The affectation of a call of a mobile in one BTS is a process which involves several parameters (ex: azimuth, tilt, power pilots) [5].

- Tilt: angle of inclination of an antenna in a vertical plan. The zone covered by the antenna decreases and the intensity of the power averages received in the cell increase.

- Azimuth: orientation of the main lobe of the antenna in the horizontal plan. A modification of the angle azimuth can be useful further to an effect of mask bound to the landscape or to the buildings (problem of interferences).

- Power of the pilots: indicate to the mobile the cell with which he has to be connected (the extent of the cell).

\subsection{General architecture of the system}

The general architecture of the system of optimization consists of various types of agents. Every agent is responsible on the resolution of a problem or the execution of a particular task. 
- Cell Agent : this agent is in charge of by the detection of the overload of a cell, through the calculation of the following function:

With:

$$
L=\frac{A}{C}
$$

L: Load with the cell.

A: Request in traffic on the cell.

C: Effective capacity of the cell.

If the load reaches the $100 \%$, the cell is declared "Applicant" otherwise it is declared "Candidate" with a degree of participation (number of communications to be able to take care). According to the dimensions of the zone to be covered communicated by GIS Agent "Geographical Information Systems", Cell Agent takes the initiative to choose the optimal cell among six nearby cells (the closest in the localization of the subscriber and which can cover the zone of overload), then calculate the necessary parameters at the level of: the azimuth, the tilt and the power of the pilots. At the end, it sends these parameters to Supervisor Agent.

Cell Agent preservation the history of the previous solutions, according to the arisen events. What will allow the re-use of the good solutions for a better and faster adaptation of the network during the future phases.

- Agent Supervisor: The role of this agent is to list every time which are the cells of type : "applicant" and "Candidate" with their degrees of participation in a blackboard (spaces out shared report). After a collaboration with Cell Agent, Supervisor Agent executes a reconfiguration automatic of the cell candidate who manages to lift the overload of the cell applicant, by the sending of the calculated parameters (azimuth, tilt, power of the pilots) at the level of BTS of the cell concerned candidate.

- GIS Agent: This agent possesses a geographical representation of the region of the cellular network (line, surface, dimensions) [8], with its various changes (in case of appearance of obstacles or new constructions).

The general architecture of the proposed system is illustrated by the present figure:

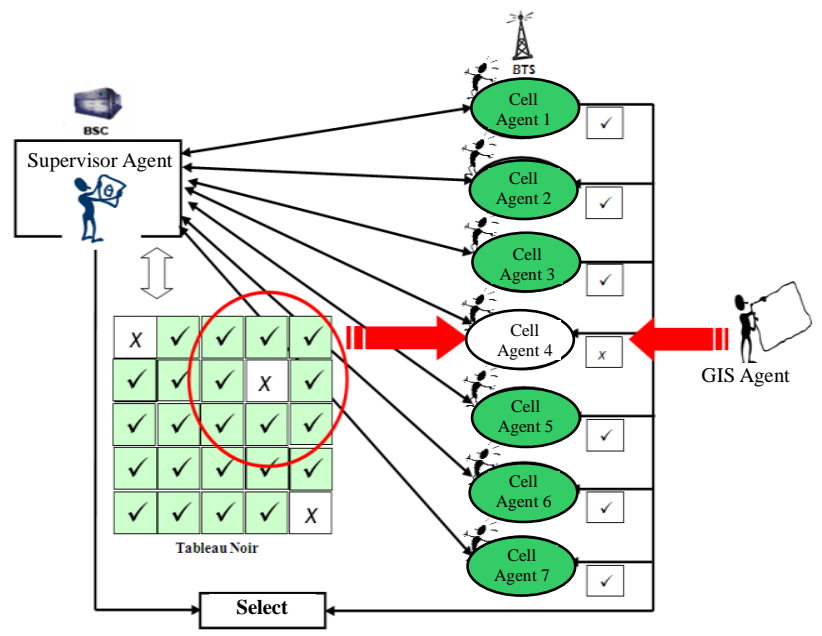

Fig 6. General architecture of the System

We can give a first prototype of Cell Agent, through this code Java (see Figure 7):

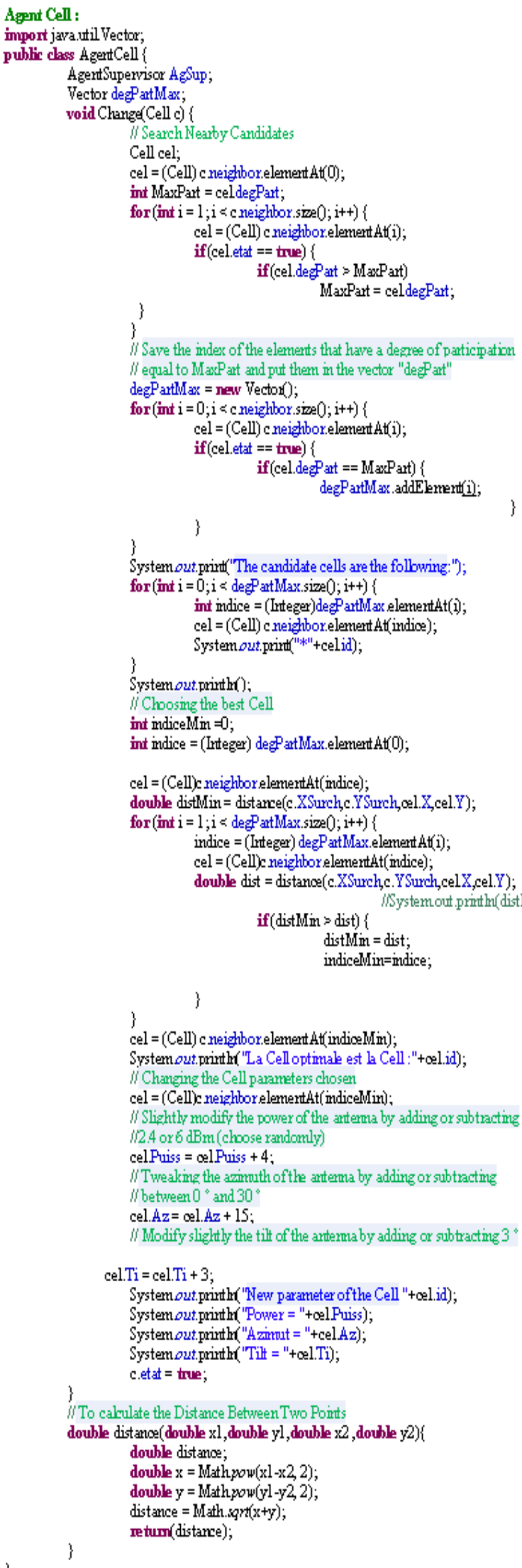

Fig 7. Prototype of Cell Agent 


\section{CONCLUSION AND PERSPECTIVES}

This paper was the object of the presentation of a new solution of a problem which enough took time researchers and which was the major concern of mobile phone operators. This solution uses a new technique, that is the multi agents systems for the implementation of a process of optimization of the cellular network. To assures revisions and recycling of the cellular network, the integration of the year intelligent agent at the level of BTS, was indispensable to increase the intelligence of this equipment. Our work is crossed by several training courses. Having spoken about the domain of the networks of the telephony and its mobile hand concepts, in particular the cellular networks, we dedicated has section for the world of the agents and their interactions. To arrives at the nucleus of our work, that was the description of the solution proposed with its hand points, in particular the presentation of the general architecture of the proposed system. As perspectives, we think that the integration of this new idea offers an advanced and simple solution in the field of the mobile telephony, and will make a new revolution, by asserting being able to apply it and adopting it in the deployment of the cellular networks and which will allow to exploit its current technical performances and to come. The gains in performance are also translated by the reduction for the operator of the investments in the infrastructure.

\section{REFERENCES}

[1] Audrey Dupont.2005. Study of a métaheuristique crosses for the affectation of frequencies in the evolutionary tactical networks. (October, 2005).

[2]Chaker MEZIOUD, M-K KHOLLADI. . 2009. In New mobile telephony operators Approach for the resolution of the problems of optimization for. Newspaper of Theoretical and Applied Information Technology. 8Vol5No3.
[3] Chaker MEZIOUD, M-K KHOLLADI. 2009. Dynamic structure for the resolution of the problems of optimization for mobile telephony operators. IEEE. GIIS.

[4] H.MABED. 2005. Study of the problems of dynamic optimization: a model for the problem of cellular evolution. University of Technology of Belfort Montbéliard.(October 2005).

[5]J. DREO, Has. Pétrowski, p. Siarry, E.Taillard. 2003 Méthaeuristiques for the difficult optimization. Edition Eyrolles.

[6] Jean-Philippe Muller. 2002. The GSM network and the mobile. Version 07/2002.

[7] M. YEKHLEF. 2007." Study of the methods of access in the mobile networks ". Magister in Electronics, option: microwaves for Telecommunication.

[8] Nadine Cullot. Christine Parent-Stefano SpaccapietraChristelle Vangenot. 2003. GIS in the geographical ontologies.

[9] P. GODLEWSKI, p. Martin, Mr Coupechoux. 1999. Cellular concepts and Parametrize Radio. (February 1999).

[10] R.JENNINGS. 1999. One agent-based engineering software. Department of electronic and Computer Science, University of Southampton, Southampton SO17 IBJ, UK Received 21 (September 99).

[11] S GIRODON. 2002 GSM, GPRS networks and UMTS. (June 2002).

[12] Y.LABROU,T.Fenin, There Peng, 1999. 'agent communication languages: the current landscape', IEEE Intelligent Systems, p 45-52, (March-April on 1999). 